\title{
Autofinanciación y crowdfunding: Nuevas vías de producción, distribución y exhibición del cine español independiente tras la crisis financiera española
}

\author{
Ciro Altabás Fernández \\ Universidad Europea de Madrid \\ ciro.altabas@uem.es
}

\begin{abstract}
Resumen:
España, a diferencia de otros países europeos, ha carecido desde los inicios de la historia del cine de una industria cinematográfica propiamente dicha, pues salvo en contados períodos, como los comprendidos entre los años 1932-36 y 1945-55'- en los que se produjo una proliferación de Estudios de cine-, no ha habido un tejido industrial consolidado que permitiera un desarrollo sostenible.

Una de las grandes debilidades de la industria la encontramos en el sector de la distribución, que ha contribuido a la falta de expansión del cine español fuera de nuestras fronteras.

Teniendo en cuenta la debilidad de la industria, muy mermada para hacer frente a la explotación de películas, es lógico pensar que si el cine comercial ha encontrado siempre dificultades en el proceso de producción, más obstáculos han tenido que superar y, aún lo afrontan en la actualidad, las producciones de bajo presupuesto, que suelen realizarse bajo un prisma más personal, por la mirada de un autor preocupado por mostrar una inquietud hacia temas más cercanos al público. Son las películas denominadas "independientes", que se realizan al margen del panorama comercial e industrial imperante.

A todo este frágil escaparate industrial del cine español se ha sumado en los últimos años las consecuencias de la crisis económica en la que está sumida el país desde el año 2008, un incremento de los recortes a la hora de la producción de proyectos que ha conducido a numerosos autores a la búsqueda de nuevas vías de financiación. La más importante y extendida es el crowdfunding, una práctica basada en el mecenazgo, en la que se ahondará en profundidad en esta investigación, al igual que en otros nuevos modos de producción, distribución y exhibición que apuestan por soluciones concretas para el desarrollo de proyectos cinematográficos low cost.

La utilización de estas nuevas vías de financiación está cambiando el panorama de la industria cultural y aún están por estudiar sus efectos a corto y largo plazo. El hallazgo de un nuevo compromiso con el público, que en modelos como el crowdfunding, se convierte en parte inversora del proyecto, revoluciona la figura tradicional del espectador con rol pasivo y alimenta nuevas y futuras relaciones económicas que dibujan un camino optimista para la industria cultural en general, y para los proyectos cinematográficos, en particular.
\end{abstract}

Palabras clave: Producción; Distribución; Exhibición; Cine independiente-Autofinanciación; Crowdfunding

\section{Self-financing and crowdfunding: New ways of production, distribution} and exhibition of independent Spanish cinema after spanish financial crisis

\begin{abstract}
:
Spain , unlike other European countries, has lacked a proper film industry since the beginning of film history because, with exception of a few periods such as those between the years 1932-36 and 1945-
\end{abstract}


55 - in which was a proliferation of cinema studies, there hasn't been a consolidated industry to allow sustainable development.

One of the great weaknesses of the industry is in the distribution sector, which has contributed to the lack of expansion of Spanish cinema outside our frontiers.

Given the weakness of the industry, it is logical to think that if commercial cinema has always found difficulties in the production process, low-budget productions planned under a more personal prism, by an author concerned with issues closer to the public, had to overcome even more obstacles. Movies that are called "independent" take place outside the prevailing commercial and industrial landscape .

Added to this fragile showcase is the economic crisis that has engulfed the country since 2008, and an increase of cuts in production has led many authors in search of new funding. The most important and widespread is the crowdfunding, a practice based on patronage in which we will deal in depth in this research, as in other new modes of production, distribution and exhibition that look for concrete solutions for project development in low-cost film.

The use of these new funding techniques is changing the cultural industry and it's still being studied in short and long term. The discovery of a new commitment from the audience, such as the crowdfunding models, that becomes part of the project investment, has revolutionized the traditional figure of the passive role viewer, and establishes future economic paths for the cultural industry in general and for film projects in particular.

Key Words: Production; Distribution; Exhibition; Independent Cinema; Financing; Crowdfunding

\section{Referencia normalizada:}

Altabás Fernández, C. (2014): Autofinanciación y crowdfunding: Nuevas vías de producción, distribución y exhibición del cine español independiente tras la crisis financiera española. Historia y Comunicación Social. Vol. 19. Núm. Especial Marzo. Págs. 387-399.

Sumario: 1. Introducción, 2. Star System de Hollywood y producción de bajo presupuesto (Low Cost.), 3. Nuevas vías de producción, distribución y exhibición del cine español independiente a partir de la crisis económica española de 2008, 3.1. La industria del cine independiente español en los años previos a la crisis económica, 3.2. La industria del cine independiente español durante la crisis económica, 3.3 Nuevos modos de producción surgidos al calor de la crisis de 2008, 3.3.1. La autofinanciación, 3.3.2. El crowdfunding, 3.4. Nuevas vías de distribución y exhibición tras la crisis de 2008.

La aplicación por parte del Ministerio de Cultural de los nuevos recortes en el sector cinematográfico el pasado año, ha supuesto un mazazo muy duro para la industria, aquejada ya de una fragilidad pomposa.

La reducción del presupuesto en un 35\% y la subida del IVA (21\%) han limitado más las posibilidades de crecimiento del cine español en general y, por tanto, también del cine "independiente", que aunque más al margen del circuito comercial, se beneficia igualmente de políticas concretas aplicadas al sector que permitirían poder llevar a cabo un proyecto cinematográfico de menor envergadura económica.

La necesidad de encontrar un flotador para evitar que definitivamente el cine se acabe ahogado, ha llevado a la búsqueda y hallazgo de nuevas vías de financiación que han ido surgiendo conforme la crisis económica iniciada en 2008 se ha ido haciendo más acuciante. 
Nuevas prácticas basadas en el mecenazgo, con la participación de micro-inversores están salvando al cine independiente de una muerte segura y están estableciendo nuevas relaciones de compromiso con el público.

De esta manera, gracias a modelos como el crowdfunding, el consumidor deja de ser un espectador pasivo para convertirse en inversor social del proyecto. "Se trata de nuevos perfiles de consumidores responsables más implicados en los procesos de producción, más exigentes ética y moralmente, sensibles socialmente y más conscientes de su fuerza"2.

Estas nuevas vías de financiación están cambiando el panorama de la industria cultural y aún están por estudiar sus efectos a corto y largo plazo. El hallazgo de un nuevo compromiso con el público, que se convierte en parte inversora del proyecto, revoluciona la figura tradicional del espectador con rol pasivo y alimenta nuevas y futuras relaciones económicas que dibujan un camino optimista para la industria cultural en general, y para los proyectos cinematográficos, en particular.

En esta investigación se tratará de realizar un análisis sobre las repercusiones del nacimiento y desarrollo de nuevas vías de producción, distribución y exhibición del cine independiente español que están contribuyendo a la supervivencia del mismo, en el marco de la crisis económica iniciada en 2008, que aún persiste.

Para realizar esta investigación se empleará una metodología analítica consistente en estudiar en profundidad la génesis, desarrollo y materialización concreta de las nuevas vías de producción, distribución y exhibición del cine independiente español así como su viabilidad en el mercado y oportunidad para impulsar la producción nacional paliando los efectos negativos de la crisis económica en la que está inmersa el país.

\section{Introducción}

Los nuevos modelos de financiación de proyectos cinematográficos buscan auxiliar a la obras de presupuesto modesto, permitiendo que puedan desarrollarse en el seno de un mercado copado por medianas y grandes producciones.

Debido a la falta de recursos económicos por parte de numerosos productores/ creadores el surgimiento de prácticas como el crowdfunding se convierten en alternativas viables para poder levantar proyectos de pequeña envergadura.

El término "crowdfunding", acuñado por Jeff Howe de la revista Wired (Ruiz Gutiérrez, 2010) hace alusión a la idea de producir de manera colectiva, es decir, de conseguir financiación a través de un conjunto de personas (mecenas) que apoyan el proyecto para sacar adelante una película, un libro, o cualquier otro producto cultural.

Además, las herramientas tecnológicas, como Internet, están facilitando la difusión de los proyectos audiovisuales, y su abaratamiento en la fase de distribución. 
De manera que la proximidad con el público es mayor y también la incidencia de las obras generadas.

\section{Star-system de Hollywood y producción de bajo presupuesto (low cost)}

Las grandes producciones por antonomasia han sido aquellas que se han gestado en los grandes estudios de Hollywood. Fue en los años 20 cuando comenzaron a forjarse las primeras grandes compañías cinematográficas en Estados Unidos (majors): la Paramount, MGM, la 20th Century Fox, la Warner Bros y RKO. A estas habría que sumar tres más pequeñas: La Universal, la Columbia y la Unites Artist (Fernández Díez.F y Martínez Abadía. J, 2010: 30).

Su época dorada fueron los años 40, cuando se filmaron las grandes producciones de los Estudios de Hollywood. Las cuatro majors producían del 60 al 75\% de las películas americanas y distribuían del 90 al 95\% de los filmes importantes (Gubern, 2006: 297).

El éxito de Hollywood residía en la especialización del trabajo y básicamente en el hecho de que los grandes directivos de las multinacionales ejercían un gran poder en la industria cinematográfica (Fernández Díez.F y Martínez Abadía. J, 2010: 24).

Las grandes productoras se traían a las mejores estrellas del star-system, apostando por las películas de género y así obteniendo el éxito garantizado. La finalidad del cine clásico de Hollywood era conseguir que el público se sintiera identificado con el héroe o protagonista, de manera que se conseguía la empatía y la adhesión del espectador lo que revertía en los beneficios de las producciones.

"Es significativo que le haya llamado la "fábrica de sueños", ya que la mayoría de películas comerciales se producen para el consumo masivo" (Kracauer, 1996:212). Y esta es precisamente una de las características del cine hollywoodiense, su conexión con los "sueños" del público.

La aparición de la televisión y su consolidación posibilitaron el crecimiento de productores independientes así como la reconversión de las grandes multinacionales, "que se afianzaron en el control de la distribución" (Fernández Díez.F y Martínez Abadía. J, 2010: 25). De manera que la industria de Hollywood se vio impulsada de nuevo en los años sesenta reforzando su dominio en el ámbito internacional hasta nuestros días.

Respecto a la producción de bajo presupuesto, se considera que el inicio de este tipo de cine "al margen de la industria" comienza a realizarse en Estados Unidos en la década de los 60 con el llamado New American Cinema Group (Gubern, 2006:355). No se trata únicamente de películas de bajo presupuesto, sino que además son filmes con un componente estético o de "independencia creativa" (Ídem). Directores como John Cassavettes o los hermanos Jonas y Adolfas Mekas, reivindican esta manera 
transgresora de hacer cine donde prima la creatividad y el ejercicio estilístico e improvisado fuera de los grandes platós de Hollywood y de sus estrellas.

La explosión del cine "indie" y buena parte de su comercialización la debemos a la figura de Quentin Tarantino que se convierte en un fenómeno de masas renovando la clásica ficción americana. Miramax, productora "indie" por excelencia "se ha convertido en una mini-mqjor que tiende a dar el soplo de muerte de lo políticamente correcto al producto americano que distribuye y a la obra de los cineastas europeos que financia cada vez con mayor asiduidad"3. Hay que decir que el cine independiente americano que surge a finales de los años ochenta plantea una mirada distinta sobre los géneros, recreando la cultura popular y su estética "pop", "trash" y "freak" (Ídem), convirtiéndose así el cine en un espejo más o menos hiperbolizado de los gustos de la sociedad.

En la década de los noventa, al tiempo que en Estados Unidos se desarrollan proyectos independientes, emergen cines "exóticos", o al menos, se revaloriza el gusto por lo asiático. Directores como el hongkonés Wong Kar Wai o el iraní, Abbas Kiarostami conviven junto a la estética más desenfadada y pop del cine norteamericano.

También es la época del Dogma 95 con su trasgresión estética, con el uso de la cámara en mano, y la propulsión del cine "doliente" a través de una temática del "pathos", que pone a prueba al más insensible de los mortales.

Lo cierto es que tanto el Dogma como el cine asiático son paradigmas de un cine de cierta resistencia y oposición a la norma establecida, aunque en algunos casos no se trata de producciones de bajo presupuesto. Sin embargo, logran evocar ese halo "indie" por su no dependencia al modo dominante o hegemónico de representación (M.R.I $\left.{ }^{4}\right)$ así como por la estética perseguida y la temática tratada.

El star system y el low cost también se diferencian en cuestiones de distribución. Ya de antemano, sin entrar en el cine independiente, entre las grandes compañías norteamericanas y las europeas existen grandes distancias.

De hecho, las empresas europeas no pueden competir con las multinacionales americanas que suelen copar el mercado de la distribución, principalmente porque al tratarse de grandes distribuidoras suelen absorber a las pequeñas, que garantizan así su supervivencia.

"La diferencia entre unas y otras distribuidoras radica en el distinto nivel comercial de las producciones que conforman sus catálogos" (Fernández Díez.F y Martínez Abadía. J, 2010: 30).

De manera que las grandes distribuidoras disponen de un variado catálogo de películas y medios publicitarios, mientras que las compañías pequeñas deben hacer una cuidada selección de las películas para garantizarse un hueco dentro del sector.

En el caso del cine independiente, muchas producciones carecen de distribuidora por lo que no pueden comercializarse por los circuitos habituales y no llegan por 
tanto a un mínimo target. Afortunadamente, muchas películas de bajo presupuesto logran visibilidad a través de Internet, gracias a distribuidoras on-line como la española Filmin.

También, algunos festivales de cine están optando por proyectar estas mini-producciones que no se exhiben en los canales convencionales para apoyar el trabajo low-cost. En España, el Festival Rizoma, que se celebra anualmente en Madrid y que cuenta con proyecciones de cine, conciertos musicales y exposiciones de arte, suele ser un buen escaparate para las películas de bajo presupuesto con un gran potencial creativo y temática "indie". De hecho, el certamen cuenta con un premio de distribución para directores noveles que no disponen de una plataforma de lanzamiento que les otorgue la visibilidad necesaria a nivel nacional e internacional.

\section{Nuevas vías de producción, distribución y exhibición del cine español inde- pendiente a partir de la crisis económica española de 2008}

3.1. La industria del cine independiente español en los años previos a la crisis económica

Hablar de cine independiente español cuando apenas se puede hablar de industria cinematográfica en España, es un asunto complejo. Primeramente, porque las productoras españolas son muy pequeñas y mayoritariamente nominativas.

Nos referimos a cine español independiente cuando aludimos a un tipo de películas que no entra en el ya exiguo circuito comercial español, normalmente, dirigidas por cineastas que montan su productora a fin de desarrollar sus proyectos personales, apuestas con una clara renovación de los códigos comerciales basados en temáticas recurrentes propias de las películas de género.

En el caso de Antonio Banderas, éste dirigió en 2006 "El camino de los ingleses", una película que fue vista en Estados Unidos en circuitos muy minoritarios, ya que en realidad, lo que buscaba el actor era la opinión de los profesionales. "Es decir, se hace una película porque se puede hacer, pero el factor público no es lo primordial, lo que interesa es contar tú historia, quitarte tus fantasmas o creer que se contribuye a una modificación de la historia para que la verdad resplandezca y el público lo sepa" (González, S., 2007). Nuestros directores, salvo en contados casos como Pedro Almodóvar, Alejandro Amenábar o Juan Antonio Bayona, no tienen salida al exterior. Las producciones más caras de nuestro cine suelen estar financiadas por televisiones privadas (Telecinco, Antena 3).

3.2. La industria del cine independiente español durante la crisis económica

En enero de 2010 tras dos meses de bloqueo, la Comisión Europea aprobaba el sistema español de ayudas al cine, dotado con 576 millones de euros hasta el 31 de 
diciembre de 2015 y que incluye medidas de apoyo a la producción y distribución de películas ${ }^{5}$. El diario económico Cinco Días, recogía en dicha noticia las declaraciones de la comisaria de Competencia Neelie Kroes, al respecto: "Felicito a las autoridades españolas por dirigir la ayuda a incrementar la diversidad cultural de las películas disponibles para el público español. Este sistema debería mejorar la visibilidad y popularidad de las películas españolas independientes, no sólo en España sino en todo el mundo, sin falsear indebidamente la competencia". Pero las quejas de los cineastas contra la Orden venían motivadas por la denuncia de que estas ayudas tan sólo beneficiaban a las grandes producciones en detrimento de las producciones independientes, y que había provocado la paralización temporal de las ayudas para 2010. En ese año se produjeron 200 largometrajes. "Del total, 49 se han realizado en régimen de coproducción con otros países, cifra muy similar a la de 2009 en que se hicieron 51 coproducciones. Este sistema de producción se afianza como medio básico para la salida de nuestro cine al mercado exterior, y para rentabilizar las grandes inversiones iniciales que se necesitan para producir un largometraje" (Bello Cuevas, 2013).

El sector del cine venía pidiendo medidas al gobierno para potenciar la industria frente a la fuerte competencia americana que alcanzaba en 2010 una cuota cercana al 70\%. En 2012 la política de recortes presupuestarios toca de lleno al sector del cine. El Fondo Nacional de Cinematografía pierde un $35 \%$ de su presupuesto ${ }^{6}$.

El Instituto de la Cinematografía y las Artes Audiovisuales, que subvenciona los festivales de San Sebastián, Málaga y Huelva, también sufre un recorte del 35\% (de 106 millones a 68,86). Convirtiéndose el cine en el sector más castigado por los Presupuestos Generales del Estado de 2012. En plena crisis económica, directores con vocación independiente como Eduardo Chapero Jackson, Jorge Dorado o Leo Siminiani han logrado sacar adelante sus respectivos proyectos cinematográficos, no sin grandes esfuerzos. En el caso de Dorado, logró financiación en Los Angeles con la productora de un expatriado, Jaume Collet Serra, para la realización de Mindscape, un thriller psicológico. Simiani logró llevar a cabo su apuesta personal, "Mapas", bajo el sello Avalon. La película conforma un autorretrato, un viaje del director a la India motivado por una crisis personal ${ }^{7}$.

\subsection{Nuevos modos de producción surgidos al calor de la crisis de 2008}

En pleno panorama de desolación para el cine español, con la reducción de las subvenciones y el incremento del IVA, los productores españoles buscan nuevas formas de sacar adelante un proyecto cinematográfico y recurren para ello al mecenazgo- ya lo hacía Buñuel en la década de los treinta del siglo pasado- o incluso intentan- los que tienen recursos para ello- acudir a la autofinanciación, desarrollando proyectos viables y sostenibles, reduciendo al máximo los costes de producción.

\subsubsection{La autofinanciación}

La autofinanciación es una de las fórmulas que están empleando algunos realizadores que han pasado de ser únicamente directores a convertirse en "hombres orquestas" 
para poder desarrollar sus proyectos cinematográficos. Con el fin de sacar adelante la película, estos cineastas tienen que reducir al máximo los costes de producción si no quieren arruinarse. Es por ello que son los abanderados del cine low cost. La mayoría de estos realizadores se pueden considerar "autores" por el hecho de que ellos mismos suelen controlar todo el proceso de producción: guión, realización y producción.

La producción implica dos funciones (Clemente Mediavilla, 2004): la económica, que significa la búsqueda de financiación para el proyecto; y la comercial: el colocar el producto en el mercado. Con todo, la autofinanciación se ha convertido en España en una de las escasas opciones para poder desarrollar un proyecto, debido fundamentalmente a las políticas de recortes en materia cinematográfica a raíz de la crisis financiera. Ello implica que el cineasta tenga que disponer de capital propio para el desarrollo de sus proyectos.

Entre estos autores destaca Carlos Vermut, director español que ha conseguido con su primer largometraje "Diamond Flash" (2012) un gran reconocimiento en los circuitos de cine independiente.

La película, que tan sólo costó 20.000 euros, se rodó con el dispositivo de vídeo de una cámara fotográfica. Autofinanciada casi en su mayoría por el propio director, el filme se proyectó en los Festivales de Sitges y Abycine y ganó el premio Rizoma de Distribución, concedido por el Festival Rizoma, consiguiendo así financiación y canales de distribución para su exhibición. "Diamond Flash" logró el record de visionados en la distribuidora on-line Filmin y acabó en varias listas "Top 10" en $2012^{8}$. Fue editada en DVD por Cameo pero no fue estrenada en ningún cine comercial.

Tras el éxito rotundo de "Diamond Flash", Carlos Vermut ha conseguido el respaldo necesario para su nueva película "Magical Girl", está preparando su segunda película, que está presupuestada en unos 500.000 euros, 470.000 euros más que su ópera prima.

Otro de los cineastas que han decidido autofinanciar sus proyectos ante la falta de recursos y las escasas subvenciones normalmente destinadas a películas más comerciales, es Norberto Ramos del Val, quien con su productora Norberfilms ha sacado adelante cuatro películas, recurriendo a la autoproducción y al crowdfunding. Su último trabajo "Summertime", que consiguió la financiación gracias a este último método a través de micro-mecenas, se estrenó el 4 de noviembre de 2012 en la joven sala independiente Artistic Metropol, y ha logrado cuadruplicar los beneficios de cualquier otra película que se proyecta en la sala.

De nuevo, los canales de distribución empleados suelen ser las distribuidoras on-line y los festivales, sin exhibición en cines comerciales.

\subsubsection{El crowdfunding}

El crowfunding ha nacido como una práctica que sin duda ha venido acompañada de la progresiva participación del público en los proyectos audiovisuales. A raíz de 
la crisis económica, esta modalidad de producción colectiva se ha convertido en un éxito para realizadores que intentan desarrollar proyectos de consiguiendo mediante este mecenazgo parte de la financiación de la película.

Lo cierto es que esta modalidad tiene el incentivo de hacer partícipe al usuario en el proceso de producción de la película, en concreto en la fase de financiación. Según sea su aportación al proyecto, el usuario conseguirá desde la copia en DVD de la película, hasta aparecer en los títulos de crédito del filme como productor asociado.

Se puede definir el crowdfunding como una estrategia basada en la creación de una convocatoria abierta a la financiación de un proyecto, habitual-mente vía Internet y redes sociales- por parte de una multitud de personas que aportan en general pequeñas cantidades, pasando a ser reconocidos como parte del mismo (Roig Telo y otros, 2012).

"El Cosmonauta" (Nico Alcalá, 2012) fue la primera película independiente española financiada mediante crowdfunding. Se trata de un largometraje que fue impulsado en España por un grupo de jóvenes relacionados con el audiovisual que integraban el colectivo Riot Cinema y que iniciaron la andadura hacia la financiación colectiva por medio del crowdfunding. Para financiar la película que comenzaron a rodar en 2011 hallaron tres vías: las aportaciones de los mecenas, la inversión privada y licencias Creative Commons.

"La comunidad de El Cosmonauta se construye a partir de una subscripción simbólica (a partir de $2 €$ ), que convierte a los usuarios en co-productores, actuando básicamente como prescriptores del proyecto"

"El Cosmonauta" fue la primera película que hizo uso de métodos no tradicionales de financiación consiguiendo un total de 320.000 euros para la producción de la película y marcando un hito en la historia del crowdfunding (Ídem).

El filme sirvió para abrir la puerta a otros proyectos cinematográficos como "O Apóstolo" (2012), primera película de animación realizada con "stop motion" pudo arrancar su producción gracias al crowdfunding, por la aportación de 610 socios.

Este nuevo modo de financiación colectiva ha propiciado el nacimiento de plataformas y páginas webs que ponen en contacto a creadores con inversores. Un ejemplo es la website de "partizipa" (www.partizipa.com) (Vialás Fernández, 2010). En cuanto a plataformas, en los últimos años han surgido numerosas en la Red. Entre ellas, My Major Company, Kickstarter, Verkami o Goteo.

Otra práctica en boga, que se aleja de los métodos tradicionales de financiación es el crowdsourcing que lleva implícito el término de co-creación. Es decir, en este caso el usuario se convierte en parte creadora del proyecto. Un ejemplo es el proyecto Life in a day, apadrinado por Ridley Scott, en colaboración con YouTu-be.

"La idea se lanzó a través de una convocatoria global para recibir vídeos grabados desde cualquier parte del mundo en un mismo día (24 de julio de 2010); a partir de ellas, el oscarizado director Kevin McDonald (The Last King of Scotland) seleccio- 
naría las más destacadas para construir un documental que reflejaría un día en la vida de la Tierra" (Roig Telo y otros, 2012)

\subsection{Nuevas vías de distribución y exhibición tras la crisis de 2008}

En cuanto al sector de distribución, más reducido que el de producción, "también presenta diferencias de dimensión empresarial: frente a grandes distribuidoras entre las que se encuentran las filiales de las "majors" americanas, se encuentran pequeñas empresas de ámbito nacional o local” (Bello Cuevas, 2013).

Sin embargo, la incorporación de la tecnología digital al mercado cinematográfico podría cambiar completamente el negocio de la distribución a nivel mundial, si las empresas españolas adoptan los acuerdos necesarios con el sector de la exhibición (Ídem).

Un ejemplo de revolución del panorama del sector de distribución en España ha sido el éxito de la película "Carmina o revienta" de Paco León, considerada la primera película que se estrenó simultáneamente en Internet, DVD, televisión y cines. ${ }^{9}$

El filme está producido por Jaleo Films con un coste de producción de 100.000 euros, gran parte de éste capitalizado. En cine, se obtuvo, "con sus 4.650 espectadores, alrededor de 35.000 euros (cifrando en una media de 8 euros la entrada); 65.500 euros gracias a los DVD (11.000 copias a 5,95 euros) y ha recaudado en Internet, tomando como precio medio 2,5 euros, 44.125 euros por 17.650 visionados ${ }^{\prime 10}$.

En lo referente a la exhibición, ya en 2010 se produce una disminución del número de salas activas. Desde 2005 se han cerrado unas 400 salas, aunque también hemos asistido al nacimiento de salas de cine independiente como la madrileña "Artistic Metropol".

\section{Conclusiones}

Modelos de financiación alternativos a los ya tradicionales buscan por medio de estrategias creativas y sociales dar salida a las producciones cinematográficas apelando a la figura del público como micro-inversor.

Estas herramientas- tales como son el crowdfunding, el crowdsourcing o la autofinanciación- proponen alternativas viables y sostenibles de producción y distribución ante las dificultades que encuentran los cineastas a la hora de sacar adelante un proyecto cinematográfico en plena crisis financiera.

La escasez de subvenciones y la política conservadora a la hora de otorgarlas, así como el incremento del IVA han propiciado la búsqueda de soluciones a través de dos armas fundamentales: la creatividad y, sobre todo, la participación activa del público como un productor más del proyecto. 
Las nuevas tecnologías también están facilitando las cosas. Ya que a los clásicos y costosos canales de distribución clásicos se les suma ahora las distribuidoras on-line como Filmin, que contribuyen a que los proyectos puedan llegar al público.

El apoyo de algunos festivales de cine independiente a proyectos "low cost" también están contribuyendo a la posibilidad de un director de continuar haciendo cine después de su debut, al ofrecerle recursos económicos y medios de distribución, que garanticen la continuidad del trabajo cinematográfico de calidad.

\section{Bibliografía}

\subsection{Libros}

FERNÁNDEZ DÍEZ. F y MARTÍNEZ ABADÍA. J. (2010): La dirección de producción para cine y televisión. Paidós. Barcelona.

GUBERN, Román. (2006): Historia del cine. Editorial Lumen. Barcelona.

KRACAUER, Siegdried. (1996): Teoría del cine. Paidós. Barcelona.

Artículos Web

BELlO CUEVAS, J. (2013) Hacer Cine en España. Cuadernos de Documentación Multimedia, Norteamérica. Disponible en: <http://revistas.ucm.es/index.php/ CDMU/article/view/41130/39342>. [Fecha de acceso: 14 abril 2013].

Carlos Vermut, ganador del premio Rizoma 2011, rueda su segundo largometraje. Rizoma Festival. Disponible en: http://www.rizomafestival.com/rizoma-filmprize-winner-carlos-vermut-set-to-shoot-2nd-feature/. [Fecha de acceso: 18 de septiembre de 2013].

Carmina o revienta, ¿un éxito de negocio real o un primer paso para normalizar Internet?. (2013) 20 minutos. Disponible en: http://www.20minutos.es/noticia/1535524/0/carmina-o-revienta/eficacia/distribucion/. [Fecha de acceso: 14 abr. 2013].

CLEMENTE MEDIAVILLA, Jorge. (2004). La información de la producción audiovisual: el "software" aplicado como método alternativo a las fórmulas de gestión clásicas (Tesis Doctoral). Universidad Complutense de Madrid. Disponible en: http://eprints.ucm.es/tesis/inf/ucm-t27327.pdf. [Fecha de acceso: 19 de septiembre de 2013].

El cine español se lleva la peor parte del recorte de los presupuestos. (2012). El Pais. Disponible en: http://cultura.elpais.com/cultura/2012/04/02/actualidad/1333395117_112099.html. [Fecha de acceso: 12 de septiembre de 2013].

GARCÍA FERNÁNDEZ, E. (ene. 2002.) En torno a algunos problemas industriales del cine español. La producción de largometrajes y cortometrajes en España. Área Abierta. Disponible en: http://revistas.ucm.es/index.php/ARAB/article/view/ ARAB0202130001A. [Fecha de acceso: 9 de septiembre de 2013]. 
GONZÁLEZ, S. S. (2007). ¿por un cine EspaÑol?. Area Abierta, (18), 1-12. Disponible en: http://www.ucm.es/BUCM/checkip.php?/docview/208168614?accountid=14514. [Fecha de acceso: 11 de septiembre de 2013].

GUTIÉRREZ-RUBÍ, A. (Mar 14, 2012). Una alternativa al crédito. Cinco Dias. Disponible en: http://0-search.proquest.com.cisne.sim.ucm.es/docview/929483410?accountid $=14514$. [Fecha de acceso: 3 de septiembre].

La comisión europea aprueba el sistema español de ayudas al cine. (2010, Jan 28). Cinco Dias. Disponible en: http://www.ucm.es/BUCM/checkip.php?/ docview/431471800?accountid=14514. [Fecha de acceso: 1 de septiembre].

RAMOS, David. (2013) Microinversores para tus proyectos: el crowdfunding se ha convertido en una buena alternativa para conseguir financiación cuando no se puede obtener por los canales tradición. Emprendedores: las claves de la economía y el éxito profesional, ISSN 1138-9702, Nº 184, 2013 , págs. 54-56. Disponible en: http://www.emprendedores.es/crear-una-empresa/crowdfunding/ crowdfunding. [Fecha de acceso: 15 de septiembre].

ROIG TELO, Antoni; SÁNCHEZ NAVARRO, Jordi; LEIBOVITZ, Talia. (2012).jESTA PELÍCULA LA HACEMOS ENTRE TODOS! Crowdsourcing y crowdfunding como prácticas colaborativas en la producción audiovisual contemporánea. Ejemplar dedicado a: El cine ante las transformaciones tecnológicas y los flujos comunicativos globales. Icono14, ISSN-e 1697-8293, Vol. 10, N 1, 2012 . Disponible en: http://dialnet.unirioja.es/servlet/articulo? codigo=3956850 . [Fecha de acceso: 9 de septiembre de 2013].

RUIZ GUTIÉRREZ, José Ramón (2010). Crowdfunding y Creative Commons: Nuevos modelos de financiación y propiedad intelectual para la producción y distribución de proyectos audiovisuales. Cuadernos de Comunicación. № 4 . Disponible en: http://cdc.escogranada.com/2010/12/crowdfunding-y-creative-commons-nuevos-modelos-de-financiacion-y-propiedad-intelectual-para-la-produccion-y-distribucion-de-proyectos-audiovisuales/. [Fecha de acceso: 15 de septiembre].

Siete saltos al largo. El cultural. Disponible en: http://www.elcultural.es/version papel/CINE/29569/7_saltos_al_largo. [Fecha de acceso: 14 abri.2013].

VIALÁS FERNÁNDEZ̄, Simón (2010). El Cosmonauta: un modelo alternativo de producción y distribución cinematográfica. Máster en Educación y Comunicación. UAB. Alfabetización mediática y culturas digitales / coord. por José Manuel Pérez Tornero. Disponible en: http://www.gabinetecomunicacionyeducacion.com/files/ adjntos/E1\%20cosmonauta $\% 20$ un $\% 20$ modelo\%20alternativo $\% 20 \mathrm{de} \% 20$ producci\%C3\%B3n\%20y\%20distribuci\%C3\%B3n\%20cinematogr\%C3\%A1fica.pdf. [Fecha de acceso: 21 de septiembre].

WEINRICHTER LÓPEZ, Antonio. (2002) Algunos hitos de los noventa: ¿A qué canon quedarse?.Secuencias: Revista de historia del cine, ISSN 1134-6795, N ${ }^{\circ}$ 16, págs. 18-31. Disponible en: http://dialnet.unirioja.es/servlet/articulo?codigo $=3222938$ [Fecha de acceso: 4 de septiembre de 2013]. 


\section{Notas}

1 GARCÍA FERNÁNDEZ, E. (ene. 2002.) En torno a algunos problemas industriales del cine español. La producción de largometrajes y cortometrajes en España. Area Abierta. Disponible en: http://revistas.ucm.es/index.php/ARAB/article/view/ARAB0202130001A. [Fecha de acceso: 9 de septiembre de 2013].

2 GUTIÉRREZ-RUBÍ, A. (Mar 14, 2012). Una alternativa al crédito. Cinco Dias. Disponible en: http://0-search.proquest.com.cisne.sim.ucm.es/docview/929483410?accountid=14514. [Fecha de acceso: 3 de septiembre].

3 WEINRICHTER LÓPEZ, Antonio. (2002) Algunos hitos de los noventa: ¿A qué canon quedarse? Secuencias: Revista de historia del cine, ISSN 1134-6795, No 16, págs. 18-31. Disponible en: http:// dialnet.unirioja.es/servlet/articulo?codigo $=3222938$. [Fecha de acceso: 4 de septiembre de 2013].

4 Modo de Representación Institucional formulado por Noël Burch.

5 La comisión europea aprueba el sistema español de ayudas al cine. (2010, Jan 28). Cinco Días. Disponible en: http//www.ucm.es/BUCM/checkip.php?/docview/431471800?accountid=14514.

6 El cine español se lleva la peor parte del recorte de los presupuestos. El País. Disponible en: http:// cultura.elpais.com/cultura/2012/04/02/actualidad/1333395117 112099.html. [Fecha de acceso: 12 de septiembre de 2013]

7 Siete saltos al largo. El cultural. Disponible en: http://www.elcultural.es/version_papel/ CINE/29569/7_saltos_al_largo. [Fecha de acceso: 14 abri.2013].

8 Carlos Vermut, ganador del premio Rizoma 2011, rueda su segundo largometraje. Rizoma Festival. Recuperado el 18 de septiembre de 2013 en: http://www.rizomafestival.com/rizoma-film-prize-winner-carlos-vermut-set-to-shoot-2nd-feature/

9 Carmina o revienta, ¿un éxito de negocio real o un primer paso para normalizar Internet?. 20 minutos. Disponible en: http://www.20minutos.es/noticia/1535524/0/carmina-o-revienta/eficacia/distribucion/. [Fecha de acceso: 14 abr. 2013].

\section{El autor}

Ciro Altabás Fernández. Licenciado en Comunicación Audiovisual por la Universidad Europea de Madrid y diplomado en estudios de Cinematografía por la London Film School, Ciro Altabás es director de cortometrajes de ficción ("Manual práctico del amigo imaginario (abreviado)", "DVD”, "Made in Japan", "Bono") y documentales ("Hobby") que han ganado más de doscientos premios en festivales nacionales e internacionales. Altabás ha trabajado como guionista en la serie de La Sexta "Qué vida más triste", y actualmente es profesor del Departamento de Comunicación Aplicada en la Universidad Europea de Madrid, mientras desarrolla su primer largometraje de ficción y su tesis doctoral. 